\title{
ANNOUNCEMENT
}

\section{Best Papers of 2014}

(C) Higher Education Press and Springer-Verlag Berlin Heidelberg 2015

Frontiers of Environmental Science \& Engineering is pleased to announce the best paper award for 2014. In 2014, we published 104 research and review papers in Volume 8 of FESE. In order to acknowledge the previous contributions and encourage more submissions, the FESE editorial board selected two distinguished papers for their profound insights into current serious environmental issues. We hope these papers will inspire and promote the innovation in the environmental science and engineering research field.

Following are the titles and abstracts of the two best papers of 2014:

Wei WEI, Shuxiao WANG, Jiming HAO, Shuiyuan CHENG. Trends of chemical speciation profiles of anthropogenic volatile organic compounds emissions in China, 2005-2020. Front. Environ. Sci. Eng. 2014, 8(1): 27-41. DOI: 10.1007/ s11783-012-0461-4

Anthropogenic volatile organic compound (VOC) emissions has adverse influence on ambient air quality, human and ecological health and agriculture production by producing tropospheric ozone via photochemical processes. This paper calculates detailed chemical profiles of China's anthropogenic VOCs emissions during 2005-2020, and estimates the trends of VOCs emissions and their impact on photochemical ozone formation using the Photochemical Ozone Creation Potential (POCP), which directly linked VOC emissions to atmospheric ozone level. The study improves our understanding in the mechanisms of air pollution complex as well as to formulate cost-effective control measures of anthropogenic VOCs.

Hongguang CHENG, Xiao PU, Yiting CHEN, Fanghua HAO, Liming DONG. Characterization of phosphorus species and modeling for its organic forms in eutrophic shallow lake sediments, North China. Front. Environ. Sci. Eng. 2014, 8 (6): 905-921. DOI: 10.1007/s11783-014-0650-4

Characterization of phosphorus species is of great significance for the study of lake eutrophication and related environmental problems. In this paper, variations of phosphorus and its species in surface sediment of Baiyangdian Lake are investigated by combining field surveys with sequential extraction and numerical calculation based on cluster analysis. The results showed that phosphorus concentrations exhibited apparent regional similarities, while labile organic phosphorus showed great seasonal variation in aerobic layer and significantly contributed to phosphorus enrichment in overlying water column. The findings and proposed mathematical model could provide an important support for limnology management and eutrophic control.

Jiming Hao and John C. Crittenden

Editors-in-Chief

March 20, 2015 